\title{
ALGEBRAIC MONODROMY AND OBSTRUCTIONS TO FORMALITY
}

\author{
STEFAN PAPADIMA ${ }^{1}$ AND ALEXANDER I. SUCIU ${ }^{2}$
}

\begin{abstract}
Given a fibration over the circle, we relate the eigenspace decomposition of the algebraic monodromy, the homological finiteness properties of the fiber, and the formality properties of the total space. In the process, we prove a more general result about iterated group extensions. As an application, we obtain new criteria for formality of spaces, and 1-formality of groups, illustrated by bundle constructions and various examples from low-dimensional topology and singularity theory.
\end{abstract}

\section{INTRODUCTION}

1.1. Formality. In his seminal paper on rational homotopy theory [25], Sullivan isolated an important topological property of spaces. A connected, finite-type CW-complex $X$ is said to be formal if its minimal model is quasi-isomorphic to $\left(H^{*}(X, \mathbb{Q}), 0\right)$; in particular, the rational homotopy type of $X$ is determined by its rational cohomology ring. Examples of formal spaces include compact Kähler manifolds [6] and complements of complex hyperplane arrangements [3].

There is a related property of groups. A finitely generated group $G$ is said to be 1formal if its Malcev completion (in the sense of Quillen [24]) is a quadratic complete Lie algebra; see for instance [20] for more details. If the first Betti number of $G$ is 0 or 1, then $G$ is 1-formal. Other examples of 1-formal groups include finitely generated Artin groups [15], finitely presented Bestvina-Brady groups [21], as well as pure welded braid groups [2]. As noted in [25], fundamental groups of formal spaces are 1-formal; thus, Kähler groups and arrangement groups belong to this class. In general, smooth complex quasiprojective varieties are not formal, but fundamental groups of complements of complex projective hypersurfaces are always 1-formal [16].

A well-known obstruction to formality is provided by the Massey products. If $X$ is a formal space, then all such products (of order 3 and higher) in $H^{*}(X, \mathbb{Q})$ vanish, up to indeterminacy. Similarly, if $G$ is a 1-formal group, all higher-order Massey products in $H^{2}(G, \mathbb{Q})$ vanish.

2000 Mathematics Subject Classification. Primary 20J05, 57M07. Secondary 20F34, 55P62.

Key words and phrases. Fibration, monodromy, formality, cohomology jumping loci, link, singularity.

${ }^{1}$ Partially supported by CNCSIS grant ID-1189/2009-2011 of the Romanian Ministry of Education and Research.

${ }^{2}$ Partially supported by NSA grant H98230-09-1-0012, and an ENHANCE grant from Northeastern University. 
1.2. Algebraic monodromy. In this note, we develop a new formality criterion, based on the interplay between three ingredients.

The first ingredient is a close connection between the algebraic monodromy action on the homology of an infinite cyclic Galois cover, and the Aomoto-Betti numbers associated to the cohomology ring of the base. This connection, established in [22] and adapted to our context in Section 2, requires no formality assumption.

A second general fact, noted by Dwyer and Fried in [11] and further refined in [23], is that the homological finiteness properties (over $\mathbb{C}$ ) of a free abelian Galois cover are controlled by the jump loci for the twisted Betti numbers of the base, with rank 1 complex coefficients.

Finally, 1-formality comes into play, by allowing an intimate relation between the Aomoto-Betti numbers and the twisted Betti numbers in degree one. This relation is based on a key result from our joint work with A. Dimca, [9].

For a group $G$, denote by $b_{i}(G):=\operatorname{dim}_{\mathbb{C}} H_{i}(G, \mathbb{C})$ its Betti numbers. Our main result (proved in Section 3) reads as follows.

Theorem 1.1. Suppose given two group extensions,

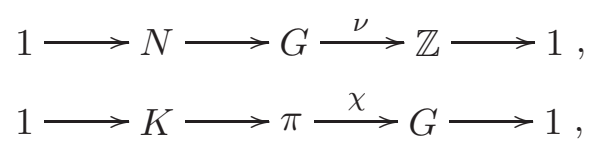

such that

(i) $b_{1}(N)<\infty$ and $b_{1}(K)<\infty$;

(ii) $\pi$ is finitely presented and 1-formal.

Then, if the monodromy action of $1 \in \mathbb{Z}$ on $H_{1}(N, \mathbb{C})$ has eigenvalue 1 , the corresponding Jordan blocks are all of size 1.

The 1-formality hypothesis is crucial here, even in the particular case (treated in Proposition 3.2) when $\pi=G$ and $\chi=\mathrm{id}_{G}$. For example, the Heisenberg group of integral, unipotent $3 \times 3$ matrices is not 1 -formal, yet it arises as an extension of $\mathbb{Z}$ by $\mathbb{Z}^{2}$, with monodromy $\left(\begin{array}{ll}1 & 1 \\ 0 & 1\end{array}\right)$.

1.3. Bundles. We illustrate Theorem 1.1 with a variety of geometric applications. To start with, we derive in Section 4 the following consequence.

Theorem 1.2. Let $U$ be a closed, connected, smooth manifold, and let $\varphi: U \stackrel{\simeq}{\longrightarrow}$ be a diffeomorphism, with mapping torus $U_{\varphi}$. Let $p: M \rightarrow U_{\varphi}$ be a locally trivial smooth fibration, with closed, connected fiber $F$. If the operator $\varphi_{*}: H_{1}(U, \mathbb{C}) \rightarrow H_{1}(U, \mathbb{C})$ has eigenvalue 1, with a Jordan block of size greater than 1 , then $M$ is not a formal space.

This theorem extends a result of Fernández, Gray, and Morgan [14], valid for bundles with fiber $F=S^{1}$. It is unclear to us whether their approach-which relies on Massey products as an obstruction to formality - still works for a general fiber $F$.

One of the inspirations for this work was the following result of Eisenbud and Neumann [13]: If $L$ is a fibered graph multilink, then its algebraic monodromy has only $1 \times 1$ Jordan 
blocks for the eigenvalue 1 . As noted by these authors, though, there do exist fibered links in $S^{3}$ for which larger Jordan blocks do occur. Necessarily, then, the complement of such a link is not formal. When combined with a result from [23] on the Bieri-Neumann-Strebel invariant, our approach yields a formality criterion for closed, orientable 3-manifolds which fiber over the circle, see Corollary 5.3.

1.4. Singularities. Given a reduced polynomial function $f: \mathbb{C}^{2} \rightarrow \mathbb{C}$ with $f(\mathbf{0})=0$ and connected generic fiber, there are two standard fibrations associated with it. One is the Milnor fibration, $S_{\epsilon}^{3} \backslash K \rightarrow S^{1}$, where $K$ is the link at the origin, for $\epsilon>0$ small. As shown by Durfee and Hain [10], the link complement is formal. Theorem 1.1 allows us then to recover the basic fact that the algebraic monodromy has no Jordan blocks of size greater than 1 for the eigenvalue $\lambda=1$.

The other fibration, $f^{-1}\left(D_{\epsilon}^{*}\right) \rightarrow D_{\epsilon}^{*}$, is obtained by "base localization." As noted in $[1,8]$, the algebraic monodromy of this fibration can have larger Jordan blocks for $\lambda=1$, see Example 6.1. It follows that the space $f^{-1}\left(D_{\epsilon}^{*}\right)$ may be non-formal. This observation is rather surprising, in view of the well-known fact that, upon localizing near $\mathbf{0} \in \mathbb{C}^{2}$ the complement of the curve $\{f=0\}$, one obtains a space homotopy equivalent to the formal space $S_{\epsilon}^{3} \backslash K$.

\section{Monodromy and the Aomoto complex}

2.1. Modules over $\mathbb{k} \mathbb{Z}$. Fix a field $\mathbb{k}$. The group-ring $\mathbb{k} \mathbb{Z}$ may be identified with the ring of Laurent polynomials in one variable, $\Lambda=\mathbb{k}\left[t^{ \pm 1}\right]$. Since $\mathbb{k}$ is a field, the ring $\Lambda$ is a principal ideal domain. Thus, every finitely-generated $\Lambda$-module $M$ decomposes into a finite direct sum of copies of $\Lambda$ (the free part), with torsion modules of the form $\Lambda /\left(f^{i}\right)$, where $f$ is an irreducible polynomial in $\Lambda$, and $i \geq 1$ is the size of the corresponding Jordan block. Define

$$
M_{f}=\bigoplus_{i \geq 1}\left(\Lambda /\left(f^{i}\right)\right)^{e_{i}(f)}
$$

to be the $f$-primary part of $M$. Clearly, the module $M_{f}$ consists of those elements of $M$ annihilated by some power of $f$. Hence, the following naturality property holds: Every $\Lambda$-linear surjection, $\phi: M \rightarrow M^{\prime}$, between finitely generated torsion $\Lambda$-modules restricts to a $\Lambda$-linear surjection, $\phi_{f}: M_{f} \rightarrow M_{f}^{\prime}$, for all irreducible polynomials $f \in \Lambda$.

2.2. Homology of $\mathbb{Z}$-covers. Let $X$ be a connected, finite-type $C W$-complex. Without loss of generality, we may assume $X$ has a single 0 -cell, say $x_{0}$. Let $G=\pi_{1}\left(X, x_{0}\right)$ be the fundamental group.

Given an epimorphism $\nu: G \rightarrow \mathbb{Z}$, let $X^{\nu} \rightarrow X$ be the Galois cover corresponding to $\operatorname{ker}(\nu)$. Denote by $\mathbb{k} \mathbb{Z}_{\nu}$ the group ring of $\mathbb{Z}$, viewed as a (right) module over $\mathbb{k} G$ via extension of scalars: $m \cdot g=m \nu(g)$, for $g \in G$ and $m \in \mathbb{k} \mathbb{Z}$. By Shapiro's Lemma, $H_{q}\left(X^{\nu}, \mathbb{k}\right)=H_{q}\left(X, \mathbb{k} \mathbb{Z}_{\nu}\right)$, as modules over $\Lambda=\mathbb{k} \mathbb{Z}=\mathbb{k}\left[t^{ \pm 1}\right]$. Since $X$ is of finite-type, $H_{q}\left(X, \mathbb{k} \mathbb{Z}_{\nu}\right)$ is a finitely generated $\Lambda$-module, for each $q \geq 0$.

Denote by $\nu_{\mathbb{Z}}$ the class in $H^{1}(X, \mathbb{Z})$ determined by $\nu$. Let $\nu_{*}: H_{1}(X, \mathbb{k}) \rightarrow H_{1}(\mathbb{Z}, \mathbb{k})=\mathbb{k}$ be the induced homomorphism. The corresponding cohomology class, $\nu_{\mathbb{k}} \in H^{1}(X, \mathbb{k})$, is 
the image of $\nu_{\mathbb{Z}}$ under the coefficient homomorphism $\mathbb{Z} \rightarrow \mathbb{k}$. We then have

$$
\nu_{\mathbb{k}} \cup \nu_{\mathbb{k}}=0 \in H^{2}(X, \mathbb{k}) .
$$

Indeed, by obstruction theory, there is a map $p: X \rightarrow S^{1}$ and a class $\omega \in H^{1}\left(S^{1}, \mathbb{Z}\right)$ such that $\nu_{\mathbb{Z}}=p^{*}(\omega)$. Hence, $\nu_{\mathbb{Z}} \cup \nu_{\mathbb{Z}}=p^{*}(\omega \cup \omega)=0$, and the claim follows by naturality of cup products with respect to coefficient homomorphisms.

As a consequence, left-multiplication by $\nu_{\mathbb{k}}$ turns the cohomology ring $H^{*}(X, \mathbb{k})$ into a cochain complex,

$$
\left(H^{*}(X, \mathbb{k}), \cdot \nu_{\mathbb{k}}\right): H^{0}(X, \mathbb{k}) \stackrel{\nu_{\mathbb{k}}}{\longrightarrow} H^{1}(X, \mathbb{k}) \stackrel{\nu_{\mathbb{k}}}{\longrightarrow} H^{2}(X, \mathbb{k}) \longrightarrow \cdots
$$

which we call the Aomoto complex of $H^{*}(X, \mathbb{k})$, with respect to $\nu_{\mathbb{k}}$. The Aomoto-Betti numbers of $X$, with respect to the cohomology class $\nu_{\mathbb{k}} \in H^{1}(X, \mathbb{k})$, are defined as

$$
\beta_{q}\left(X, \nu_{\mathbb{k}}\right):=\operatorname{dim}_{\mathbb{k}} H^{q}\left(H^{*}(X, \mathbb{k}), \cdot \nu_{\mathbb{k}}\right) .
$$

In [22], we defined a spectral sequence, starting at $E_{s, t}^{1}\left(X, \mathbb{k} \mathbb{Z}_{\nu}\right)$, and converging to $H_{s+t}\left(X, \mathbb{k} \mathbb{Z}_{\nu}\right)$. In the process, we investigated the monodromy action of $\mathbb{Z}$ on $H_{*}\left(X, \mathbb{k} \mathbb{Z}_{\nu}\right)$, relating the triviality of the action to the exactness of the Aomoto complex defined by $\nu_{\mathbb{k}} \in H^{1}(X, \mathbb{k})$, as follows.

Theorem 2.1 ([22]). For each $k \geq 0$, the following are equivalent:

(1) For each $q \leq k$, the $\mathbb{k} \mathbb{Z}$-module $H_{q}\left(X, \mathbb{k}_{\nu}\right)$ is finite-dimensional over $\mathbb{k}$, and contains no $(t-1)$-primary Jordan blocks of size greater than 1 .

(2) $\beta_{0}\left(X, \nu_{\mathbb{R}}\right)=\cdots=\beta_{k}\left(X, \nu_{\mathbb{k}}\right)=0$.

2.3. Kernels of epimorphisms to $\mathbb{Z}$. Similar considerations apply to groups. Let $G$ be a group, and let $\nu: G \rightarrow \mathbb{Z}$ be an epimorphism with kernel $N$. Let $X=K(G, 1)$ be a classifying space for $G$. Then the monodromy action of $\Lambda=\mathbb{k}\left[t^{ \pm 1}\right]$ on $H_{1}\left(X, \mathbb{k}_{\nu}\right)=$ $H_{1}(N, \mathbb{k})$ is induced by conjugation: the generator $t \in \Lambda$ acts on $H_{1}(N, \mathbb{k})$ by $\left(\iota_{g}\right)_{*}$, where $g \in G$ is such that $\nu(g)=1$ and $\iota_{g}$ is the corresponding inner automorphism.

Clearly, the Aomoto complex $\left({ }^{*}\right)$ depends in this case only on $G$ and $\nu_{\mathbb{k}}$; thus, we may denote $\beta_{q}\left(X, \nu_{\mathbb{k}}\right)$ by $\beta_{q}\left(G, \nu_{\mathbb{k}}\right)$. In the sequel, we will need the following group-theoretic version of Theorem 2.1.

Corollary 2.2. Let $1 \rightarrow N \rightarrow G \stackrel{\nu}{\rightarrow} \mathbb{Z} \rightarrow 1$ be a group extension, with $G$ finitely presented. Then, the following are equivalent:

(1) The $\mathbb{k} \mathbb{Z}$-module $H_{1}(N, \mathbb{k})$ is finite-dimensional over $\mathbb{k}$, and contains no $(t-1)$ primary Jordan block of size greater than 1 .

(2) $\beta_{1}\left(G, \nu_{\mathbb{k}}\right)=0$.

Proof. Let $X$ be a finite presentation 2-complex for $G$. Note that

$$
H_{0}\left(X, \Lambda_{\nu}\right)=\Lambda /(t-1) \text { and } \beta_{0}\left(X, \nu_{\mathbb{R}}\right)=0 \text {. }
$$

Applying Theorem 2.1 to $X$, for $k=1$, we find that $H_{1}\left(X, \Lambda_{\nu}\right)$ is finite-dimensional over $\mathbb{k}$, with trivial monodromy action on the $(t-1)$-primary part, if and only if $\beta_{1}\left(X, \nu_{\mathbb{k}}\right)=0$. But $H_{1}\left(X, \Lambda_{\nu}\right)=H_{1}(N, \mathbb{k})$ and $\beta_{1}\left(X, \nu_{\mathbb{k}}\right)=\beta_{1}\left(G, \nu_{\mathbb{k}}\right)$, and we are done. 


\section{Monodromy AND FORMALity}

This section is devoted to proving Theorem 1.1 from the Introduction. We first prove the special case in which $\pi=G$ and $\chi=\mathrm{id}_{G}$, and then reduce the general case to the special one, by means of two lemmas.

3.1. A special case. We start by recalling a result (Corollary 6.7 from [23]), relating the homological finiteness properties of certain normal subgroups of a 1-formal group to the vanishing of the corresponding Aomoto-Betti numbers.

Theorem 3.1 ([23]). Let $G$ be a finitely generated, 1-formal group. Suppose $\nu: G \rightarrow \mathbb{Z}$ is an epimorphism, with kernel $N$. Then $b_{1}(N)<\infty$ if and only if $\beta_{1}\left(G, \nu_{\mathbb{C}}\right)=0$.

We are now ready to prove an important particular case of Theorem 1.1.

Proposition 3.2. Let $G$ be a finitely presented, 1-formal group, and let $\nu: G \rightarrow \mathbb{Z}$ be an epimorphism, with kernel $N$. If $b_{1}(N)<\infty$, then the eigenvalue 1 of the monodromy action of $1 \in \mathbb{Z}$ on $H_{1}(N, \mathbb{C})$ has only $1 \times 1$ Jordan blocks.

Proof. Since $G$ is 1 -formal and $b_{1}(N)<\infty$, Theorem 3.1 insures that $\beta_{1}\left(G, \nu_{\mathbb{C}}\right)=0$. The conclusion follows from Corollary 2.2 , for $\mathbb{k}=\mathbb{C}$.

3.2. Two lemmas. Before proceeding, recall the extensions $\left(\xi_{\nu}\right)$ and $\left(\xi_{\chi}\right)$ from Section 1.2. Set $\Gamma:=\operatorname{ker}(\nu \circ \chi)$. We then have an extension

$$
1 \longrightarrow \Gamma \longrightarrow \pi \stackrel{\nu \circ \chi}{\longrightarrow} \mathbb{Z} \longrightarrow 1
$$

Pulling back $\left(\xi_{\chi}\right)$ to $N$ gives rise to another extension,

$$
1 \longrightarrow K \longrightarrow \Gamma \stackrel{\eta}{\longrightarrow} N \longrightarrow 1 \text {. }
$$

The first lemma is a standard application of the Hochschild-Serre spectral sequence of a group extension, as recorded for instance in Brown [4, p. 171]. For the sake of completeness, we include a proof.

Lemma 3.3. If both $b_{1}(N)$ and $b_{1}(K)$ are finite, so is $b_{1}(\Gamma)$.

Proof. Consider the spectral sequence of extension $\left(\xi_{\eta}\right)$, with $\Gamma$-trivial coefficients $\mathbb{C}$ :

$$
E_{s t}^{2}=H_{s}\left(N, H_{t}(K, \mathbb{C})\right) \Rightarrow H_{s+t}(\Gamma, \mathbb{C}) .
$$

Clearly, $E_{01}^{2}=H_{1}(K, \mathbb{C})_{N}$ is a quotient of $H_{1}(K, \mathbb{C})$, and $E_{10}^{2}=H_{1}(N, \mathbb{C})$. Thus, both $E_{01}^{2}$ and $E_{10}^{2}$ are finite-dimensional over $\mathbb{C}$. Hence, $H_{1}(\Gamma, \mathbb{C})$ is also finite-dimensional.

The second lemma shows that the Jordan blocks of size greater than 1 for the eigenvalue 1 propagate from $\left(\xi_{\nu}\right)$ to $\left(\xi_{\nu \chi}\right)$. As usual, write $\Lambda=\mathbb{C} \mathbb{Z}=\mathbb{C}\left[t^{ \pm 1}\right]$.

Lemma 3.4. If the $\Lambda$-module $H_{1}(\Gamma, \mathbb{C})_{t-1}$ is trivial, then so is the module $H_{1}(N, \mathbb{C})_{t-1}$. 
Proof. Pick $g \in G$ such that $\nu(g)=1$, and then $x \in \pi$ such that $\chi(x)=g$. Clearly, $\iota_{g} \circ \eta=\eta \circ \iota_{x}$. It follows that the surjection

$$
\eta_{*}: H_{1}(\Gamma, \mathbb{C}) \rightarrow H_{1}(N, \mathbb{C})
$$

is $\Lambda$-linear with respect to the corresponding monodromy actions.

Now, since both $H_{1}(\Gamma, \mathbb{C})$ and $H_{1}(N, \mathbb{C})$ are finitely generated, torsion $\Lambda$-modules, we infer from the discussion in Section 2.1 that $\eta$ induces a $\Lambda$-linear surjection on $(t-1)$ primary parts,

$$
\left(\eta_{*}\right)_{t-1}: H_{1}(\Gamma, \mathbb{C})_{t-1} \rightarrow H_{1}(N, \mathbb{C})_{t-1}
$$

The claim follows.

3.3. Proof of Theorem 1.1. The finite-dimensionality assumption (i), together with Lemma 3.3 imply that $b_{1}(\Gamma)<\infty$.

Suppose that the monodromy action on $H_{1}(N, \mathbb{C})$ coming from extension $\left(\xi_{\nu}\right)$ has eigenvalue 1, with a Jordan block of size greater than 1. By Lemma 3.4, the same holds for the monodromy action on $H_{1}(\Gamma, \mathbb{C})$, coming from extension $\left(\xi_{\nu \chi}\right)$.

Finally, we use the formality assumption (ii). From Proposition 3.2, applied to extension $\left(\xi_{\nu \chi}\right)$, we deduce that all Jordan blocks of the monodromy action on $H_{1}(\Gamma, \mathbb{C})$ have size 1 . But this is a contradiction, and the proof is finished.

\section{Bundle constructions}

Let $U$ be a closed, connected, smooth manifold, and let $\varphi: U \stackrel{\simeq}{\longrightarrow} U$ be a diffeomorphism, with mapping torus $U_{\varphi}$. Let $p: M \rightarrow U_{\varphi}$ be a locally trivial smooth fibration, with closed, connected fiber $F$. Theorem 1.2 follows from the next proposition.

Proposition 4.1. If the operator $\varphi_{*}: H_{1}(U, \mathbb{C}) \rightarrow H_{1}(U, \mathbb{C})$ has eigenvalue 1 , with a Jordan block of size greater than 1 , then $\pi_{1}(M)$ is not a 1-formal group.

Proof. The homotopy exact sequence of the mapping torus fibration, $U \hookrightarrow U_{\varphi} \rightarrow S^{1}$, yields an extension of fundamental groups, $1 \rightarrow N \rightarrow G \rightarrow \mathbb{Z} \rightarrow 1$. Clearly, $b_{1}(N)=$ $b_{1}(U)<\infty$, and the monodromy action of $1 \in \mathbb{Z}$ on $H_{1}(N, \mathbb{C})$ coincides with $\varphi_{*}$.

Similarly, the fibration $F \hookrightarrow M \stackrel{p}{\rightarrow} U_{\varphi}$ yields an epimorphism on fundamental groups, $p_{\sharp}: \pi \rightarrow G$. The group $K:=\operatorname{ker}\left(p_{\sharp}\right)$ is a quotient of $\pi_{1}(F)$; hence, $b_{1}(K)<\infty$.

Plainly, the group $\pi=\pi_{1}(M)$ is finitely presented. Given the assumption on $\varphi_{*}$, Theorem 1.1 implies that $\pi$ is not 1 -formal.

For a class $b \in H^{*}(U, \mathbb{Z})$, denote by $b_{\mathbb{R}}$ its image in $H^{*}(U, \mathbb{R})$. Proposition 4.1 gives a substantial extension of the following result of Fernández, Gray, and Morgan, contained in Proposition 9 and Note 10 from [14].

Theorem 4.2 ([14]). Let $\varphi$ be an orientation-preserving diffeomorphism of a closed, connected, oriented manifold $U$. Suppose there is an element $b \in H^{1}(U, \mathbb{Z})$ with $\varphi^{*}(b)=$ $b$, such that $0 \neq b_{\mathbb{R}} \in \operatorname{im}\left(\varphi^{*}-\mathrm{id}\right)$. If $E \rightarrow U_{\varphi}$ is a circle bundle, then $E$ is not formal. 


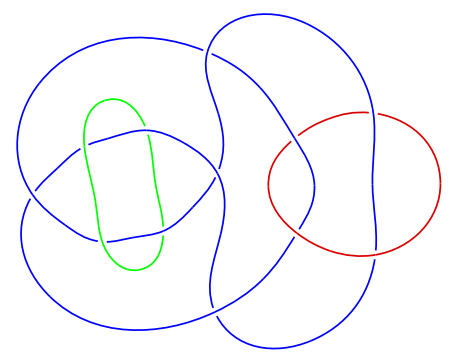

Figure 1. Eisenbud and Neumann's 3-component link

Remark 4.3. When $U$ is a symplectic manifold, and $\varphi$ is a symplectic diffeomorphism, the circle bundle $E \rightarrow U_{\varphi}$ from above can be chosen so that $E$ carries a symplectic structure [14, Theorem 4]. Yet, by Theorem 4.2 and the main result of [6], the manifold $E$ admits no Kähler metric.

In a similar vein, the particular case of Theorem 1.1 proved in Section 3 has the following geometric counterpart.

Corollary 4.4. Let $F \hookrightarrow X \rightarrow S^{1}$ be a smooth fibration, with monodromy $h$. Assume the fiber $F$ is connected and has the homotopy type of a $C W$-complex with finite 2-skeleton. If the algebraic monodromy, $h_{*}: H_{1}(F, \mathbb{C}) \rightarrow H_{1}(F, \mathbb{C})$, has eigenvalue 1 , with a Jordan block of size greater than 1 , then the group $G=\pi_{1}(X)$ is not 1-formal.

Proof. Let $1 \rightarrow N \rightarrow G \rightarrow \mathbb{Z} \rightarrow 1$ be the extension of fundamental groups determined by the fibration. From the hypothesis on the fiber $F$, the group $G$ is finitely presented, and $b_{1}(N)<\infty$. The conclusion follows from Proposition 3.2.

\section{LINKS AND CLOSED 3-MANIFOLDS}

5.1. Links in the 3-sphere. The question of deciding whether a link complement is formal has a long history. In fact, the higher-order products were defined by W. Massey precisely for detecting higher-order linking phenomena for links such as the Borromean rings.

The following example shows how the non-formality of a fibered link complement can be detected by the algebraic monodromy. It also shows that the 1-formality hypothesis is essential in Theorem 1.1.

Example 5.1. Let $L$ be the 3-component link in $S^{3}$ depicted in Figure 1. ${ }^{1}$ As explained in [13, Example 11.6], the link exterior, $X=S^{3} \backslash\left(L \times \operatorname{int} D^{2}\right)$, fibers over $S^{1}$, with fiber $F$

\footnotetext{
${ }^{1}$ This picture was drawn with the help of the Mathematica package KnotTheory, by Dror Bar-Natan, and the graphical package Knotilus, by Ortho Flint and Stuart Rankin.
} 
a connected, compact surface, and with algebraic monodromy having two Jordan blocks of type $\left(\begin{array}{ll}1 & 1 \\ 0 & 1\end{array}\right)$. By Corollary 4.4, the group $\pi_{1}(X)$ is not 1 -formal.

5.2. 3-manifolds fibering over the circle. Turning now to closed 3-manifolds, let us first recall the following result from [23], based on the interplay between the BieriNeumann-Strebel invariant, $\Sigma^{1}(G)$, and the (first) resonance variety, $\mathcal{R}^{1}(G):=\{z \in$ $\left.\operatorname{Hom}(G, \mathbb{R}) \mid \beta_{1}(G, z)>0\right\}$, of a 1-formal group $G$.

Proposition 5.2 ([23]). Let $M$ be a closed, orientable 3-manifold which fibers over the circle. If $b_{1}(M)$ is even, then $\pi_{1}(M)$ is not 1 -formal.

Combining this result with Corollary 4.4 puts strong restrictions on the algebraic monodromy of a fibration $M^{3} \rightarrow S^{1}$.

Corollary 5.3. Let $M$ be a closed, orientable 3-manifold with 1-formal fundamental group. Suppose $M$ fibers over the circle, and the algebraic monodromy has 1 as an eigenvalue. Then, there are an even number of $1 \times 1$ Jordan blocks for this eigenvalue, and no higher size Jordan blocks.

Proof. By Corollary 4.4, the algebraic monodromy has only $1 \times 1$ Jordan blocks for the eigenvalue 1 . Let $m$ be the number of such blocks. From the Wang sequence of the fibration, we deduce that $b_{1}(M)=m+1$. By Proposition 5.2, $m$ must be even.

\section{Singularities And Fibrations}

6.1. Milnor fibration. Let $f:\left(\mathbb{C}^{n+1}, \mathbf{0}\right) \rightarrow(\mathbb{C}, 0)$ be a reduced polynomial map. The link of $f$ at $\mathbf{0}$, denoted $K$, is the intersection of the hypersurface $\{f=0\}$ with the sphere $S_{\epsilon}^{2 n+1}=\left\{x \in \mathbb{C}^{n+1}|| x \mid=\epsilon\right\}$, for sufficiently small $\epsilon>0$. As shown by Milnor in [19], the map

$$
p: S_{\epsilon}^{2 n+1} \backslash K \rightarrow S^{1}, \quad p(x)=f(x) /|f(x)|
$$

is a smooth fibration. Moreover, the Milnor fiber $F=p^{-1}(1)$ has the homotopy type of an $n$-dimensional finite CW-complex. Let $\varphi: F \rightarrow F$ be the monodromy map. As is well known, the monodromy operator, $\varphi_{*}: H_{q}(F, \mathbb{C}) \rightarrow H_{q}(F, \mathbb{C})$, has only roots of unity as eigenvalues, with corresponding Jordan blocks of size at most $q+1$, see for instance [7, p. 75].

In the case when $f$ has an isolated singularity at $\mathbf{0}$, more can be said. First of all, as shown by Milnor, the fiber $F$ is $(n-1)$-connected. Moreover, $\varphi_{*}: H_{n}(F, \mathbb{C}) \rightarrow H_{n}(F, \mathbb{C})$ has Jordan blocks of size at most $n$ for the eigenvalue 1 , see for instance [12].

Particularly tractable is the case of a reduced polynomial map $f: \mathbb{C}^{2} \rightarrow \mathbb{C}$ with $f(\mathbf{0})=$ 0 , when $\mathbf{0}$ is necessarily an isolated singularity. As shown by Durfee and Hain in [10, Theorem 4.2], the link complement, $X=S_{\epsilon}^{3} \backslash K$, is a formal space. Corollary 4.4, then, explains from a purely topological point of view why the Jordan blocks of $\varphi_{*}: H_{1}(F, \mathbb{C}) \rightarrow$ $H_{1}(F, \mathbb{C})$ corresponding to the eigenvalue 1 must all have size 1. 
6.2. Base localization. Let $f: \mathbb{C}^{2} \rightarrow \mathbb{C}$ be a polynomial map with connected generic fiber. For $\epsilon>0$, there is a fibration $f: f^{-1}\left(D_{\epsilon}^{*}\right) \rightarrow D_{\epsilon}^{*}$ induced by $f$, where $D_{\epsilon}^{*}=\{t \in$ $\mathbb{C}|0<| t \mid<\epsilon\}$; the topological type of this fibration is independent of small $\epsilon$.

Now let $F$ be the generic fiber of $f$, and let $\varphi: F \rightarrow F$ be the monodromy of the fibration. Note that $F$ is a finite $\mathrm{CW}$-complex, up to homotopy; see for instance [7, p. 27]. It was shown in $[1,8]$ that $\varphi_{*}: H_{1}(F, \mathbb{C}) \rightarrow H_{1}(F, \mathbb{C})$ can have a Jordan block of size 2 for the eigenvalue 1 . The following concrete examples were communicated to us by Alex Dimca.

Example 6.1. Consider the polynomial $f=x^{3}+y^{3}+x y$. In this case, the central fiber $F_{0}=f^{-1}(0)$ has just a node singularity. Using Theorem 1 or Corollary 6 from [1], we see that $\varphi_{*}: H_{1}(F, \mathbb{C}) \rightarrow H_{1}(F, \mathbb{C})$ has a Jordan block of type $\left(\begin{array}{ll}1 & 1 \\ 0 & 1\end{array}\right)$.

Other examples are suitable translates of the polynomials $f_{B}$ and $f_{C}$ introduced in [8, Example 4], such that the central fiber corresponds to the Kodaira types $I_{1}$ or $I_{2}$. In this case, the central fibers are smooth, but the non-triviality of the monodromy is due to singularities at infinity. The polynomial $f_{B}$ (known as the Briançon polynomial) is also considered in Example 5 from [1].

Further examples can be found in [18]. In all such examples, we conclude from Corollary 4.4 that the group $\pi_{1}\left(f^{-1}\left(D_{\epsilon}^{*}\right)\right)$ is not 1 -formal.

For a non-constant polynomial map, $f: \mathbb{C}^{2} \rightarrow \mathbb{C}$ with $f(\mathbf{0})=0$, let $V=\{f=0\}$ be the corresponding plane algebraic curve, and $Y=\mathbb{C}^{2} \backslash V$ its complement. In recent work, Cogolludo-Agustin and Matei [5], and Macinic [17], proved, by different methods, that $Y$ is a formal space. Due to the local conic structure of affine varieties (see for instance [7, p. 23]), for small enough $\delta>0$, the intersection

$$
Y \cap\left\{x \in \mathbb{C}^{2}|| x \mid \leq \delta\right\}
$$

has the same homotopy type as the link complement $S_{\epsilon}^{3} \backslash K$. In conclusion, the formality property of $Y$ is preserved by localization near $\mathbf{0} \in \mathbb{C}^{2}$, but it may be destroyed by base localization, that is, when replacing the complement $Y$ by

$$
Y \cap f^{-1}\left(\left\{t \in \mathbb{R E F}^{\mathrm{E}}|\mathrm{t}| \mathrm{ENCES}_{\epsilon}\right\}\right)=f^{-1}\left(D_{\epsilon}^{*}\right) .
$$

[1] E. Artal Bartolo, P. Cassou-Noguès, A. Dimca, Sur la topologie des polynômes complexes, in: Singularities (Oberwolfach, 1996), pp. 317-343, Progress in Math. vol. 162, Birkhäuser, Basel, 1998. MR1652480 1.4, 6.2, 6.1

[2] B. Berceanu, S. Papadima, Universal representations of braid and braid-permutation groups, J. Knot Theory Ramifications 18 (2009), no. 7, 999-1019. 1.1

[3] E. Brieskorn, Sur les groupes de tresses, in: Séminaire Bourbaki, 1971/72, Lect. Notes in Math. 317, Springer-Verlag, 1973, pp. 21-44. MR0422674 1.1

[4] K. S. Brown, Cohomology of groups, Grad. Texts in Math., vol. 87, Springer-Verlag, New YorkBerlin, 1982. MR0672956 3.2

[5] J. I. Cogolludo-Agustin, D. Matei, Cohomology algebra of plane curves, weak combinatorial type, and formality, preprint arxiv:0711.1951. 6.2

[6] P. Deligne, P. Griffiths, J. Morgan, D. Sullivan, Real homotopy theory of Kähler manifolds, Invent. Math. 29 (1975), no. 3, 245-274. MR0382702 1.1, 4.3

[7] A. Dimca, Singularities and topology of hypersurfaces, Universitext, Springer-Verlag, New York, 1992. MR1194180 6.1, 6.2, 6.2 
[8] A. Dimca, Monodromy at infinity for polynomials of two variables, J. Algebraic Geom. 7 (1998), no. 4, 771-779. MR1642749 1.4, 6.2, 6.1

[9] A. Dimca, S. Papadima, A. Suciu, Topology and geometry of cohomology jump loci, Duke Math. Journal 148 (2009), no. 3, 405-457. 1.2

[10] A. Durfee, R. Hain, Mixed Hodge structures on the homotopy of links, Math. Ann. 280 (1988), no. 1, 69-83; MR0928298 1.4, 6.1

[11] W. G. Dwyer, D. Fried, Homology of free abelian covers. I, Bull. London Math. Soc. 19 (1987), no. 4, 350-352. MR0887774 1.2

[12] W. Ebeling, Monodromy, in: Singularities and computer algebra, pp. 129-155, London Math. Soc. Lecture Note Ser., vol. 324, Cambridge Univ. Press, Cambridge, 2006. MR2228229 6.1

[13] D. Eisenbud, W. Neumann, Three-dimensional link theory and invariants of plane curve singularities, Annals of Math. Studies, vol. 110, Princeton University Press, Princeton, NJ, 1985. MR0817982 $1.3,5.1$

[14] M. Fernández, A. Gray, J. Morgan, Compact symplectic manifolds with free circle actions, and Massey products, Michigan Math. J. 38 (1991), no. 2, 271-283. MR1098863 1.3, 4, 4.2, 4.3

[15] M. Kapovich, J. Millson, On representation varieties of Artin groups, projective arrangements and the fundamental groups of smooth complex algebraic varieties, Inst. Hautes Études Sci. Publ. Math. 88 (1998), 5-95. MR1733326 1.1

[16] T. Kohno, On the holonomy Lie algebra and the nilpotent completion of the fundamental group of the complement of hypersurfaces, Nagoya Math. J. 92 (1983), 21-37. MR0726138 1.1

[17] A. Macinic, Cohomology rings and formality properties of nilpotent groups, preprint arxiv:0801.4847. 6.2

[18] F. Michel, C. Weber, On the monodromies of a polynomial map from $\mathbb{C}^{2}$ to $\mathbb{C}$, Topology 40 (2001), no. 6, 1217-1240. MR1867243 6.2

[19] J. W. Milnor, Singular points of complex hypersurfaces, Ann. of Math. Studies, no. 61 Princeton Univ. Press, Princeton, N.J., 1968. MR0239612 6.1

[20] S. Papadima, A. Suciu, Chen Lie algebras, International Math. Research Notices 2004 (2004), no. 21, 1057-1086. MR2037049 1.1

[21] S. Papadima, A. Suciu, Algebraic invariants for Bestvina-Brady groups, J. London Math. Society, 76 (2007), no. 2, 273-292. MR2363416 1.1

[22] S. Papadima, A. Suciu, The spectral sequence of an equivariant chain complex and homology with local coefficients, preprint arxiv:0708.4262, to appear in Trans. Amer. Math. Soc. 1.2, 2.2, 2.1

[23] S. Papadima, A. Suciu, Bieri-Neumann-Strebel-Renz invariants and homology jumping loci, preprint arxiv:0812.2660 1.2, 1.3, 3.1, 3.1, 5.2, 5.2

[24] D. Quillen, Rational homotopy theory, Ann. of Math. 90 (1969), no. 2, 205-295. MR0258031 1.1

[25] D. Sullivan, Infinitesimal computations in topology, Inst. Hautes Études Sci. Publ. Math. 47 (1977), 269-331. MR0646078 1.1

Institute of Mathematics Simion Stoilow, P.O. Box 1-764, RO-014700 Bucharest, Romania E-mail address: Stefan.Papadima@imar.ro

Department of Mathematics, Northeastern University, Boston, MA 02115, USA

E-mail address: a.suciu@neu.edu

$U R L:$ http://www. math.neu.edu/ ${ }^{\sim}$ suciu 